\title{
Drei Fälle von Hernia diaphragmatica congenita.
}

\author{
Von
}

\author{
Dr. B is ch of f \\ in Erlangen. \\ (Mit 4 Holzschnitten.)
}

Durch die Güte meines Chefs, des Herrn Prof. Zw eifel, sind drei Fälle von angeborener Zwerchfellshernie zu meiner Kenntniss gelangt, deren Mittheilung mir einiges Interesse $\mathrm{zu}$ bieten scheint.

Es ist nicht meine Absicht, eine Zusammenstellung möglichst vieler Fälle zu veranstalten, zumal dies schon $\mathrm{Kohn}$ und $\mathrm{Lach}$ er in ausgedehntem Maasse gethan haben, vielmehr möchte ich versuchen, durch Vergleichung der drei Fälle mit anderen congenitalen $Z_{w}$ erchfellshernien eine gewisse Gleichartigkeit in der Art der Entstehung derselben nachzuweisen.

Sehr zu statten kam mir in diesem Bestreben die jüngst in der "Deutschen medicinischen Wochenschrift" veröffentlichte Arbeit Waldeyer's über die Entstehung des Diaphragma, über welchen Vorgang meines Wissens eine genauere Angabe noch nicht gemacht worden war.

Der erste der drei Fälle betrifft ein ausgetragenes Kind männlichen Geschlechtes, das in der geburtshülflichen Klinik des Herrn Professor Gusserow in Strassburg geboren wurde. Herrn Professor $\mathrm{Z}$ weifel verdanke ich die Mittheilung der näheren Umstände während der Zeit der Schwangerschaft und während der Geburt.

Schon während der Schwangerschaft war eine Zwillingsschwangerschaft zwar vermuthet, aber die sichere Diagnose derselben nicht gestellt worden, da die starke Ausdehnung des 'Leibes und die auffallend starke Fluctuation ebenso gut von $\mathrm{Hy}$ dramnion herrühren konnte und der Nachweis zweier kindlicher Herztöne nicht gelang. 
Bei der schnell verlaufenden Geburt bestätigte sich die Vermuthung der Zwillingsschwangerschaft. Das zuerst geborene Kind machte sofort eine kräftige Inspirationsbewegung, doch folgte erst nach einer längeren Pause eine zweite, oberflächliche. Dann blieben die Respirationsbewegungen vollständig aus, und auch nach energisehen Wiederbelebungsversuchen gelang es nicht, das Kind $z \mathfrak{u}$ weiteren Athembewegungen $z \mathfrak{u}$ bringen; es blieb das Rind asphyktisch. Der Herzspitzenstoss konnte noch einige Zeit lang und $z$ war im rechten Thoraxraume dicht unter der Clavicula gefuihlt werden. Nach einer halben Stunde erlosch auch die Herzthätigkeit.

Auch das zweite Kind, ein Knabe, wurde asphyktisch geboren. Die Belebung gelang bald, doch starb es am elften Tage an Lebensschwäche. Die Section ergab nichts Besonderes.

Anders war der Sectionsbefund der ersten, bald nach der Geburt geöffneten Kindesleiche.

Die äussere Inspection ergab einen durchaus normalen, die innere folgenden Befund: Die Bauchhöhle war zum weitaus grössten Theile von der ungemein stark entwickelten Leber ausgefüllt und enthielt ausser derselben nur noch die mit dem Nabel durch einen breiten bindegewebigen Strang zusammenhängende Blase, die beiden Nieren und von dem Darmkanale nur das Rectum, S-romanum und eine kurze Schlinge des Colon descendens. Alle übrigen Bauchorgane waren durch einen langen, nicht eben sehr breiten Schlitz in dem hinteren Theile der linken Zwerchfellshälfte in den Brustraum ausgetreten.

Der normale Inhalt der Brusthöhle war hierdurch vollständig in der Weise aus seiner Lage gedrängt, dass die linke, nur rudimentär gebildete Lunge vollständig nach hinten und innen gedrängt war; an ihrer Vorderfläche war eine seichte Furche als Andeutung der Lappentrennung vorhanden, hinten dagegen erschien sie vollständig glatt. Das Herz mit dem Herzbeutel war vollständig in die rechte Brusthälfte verdrängt, demselben anliegend und nach vorn das Herz überragend lag die comprimirte, nur ganz wenig lufthaltige rechte Lunge, an der normalen Stelle die Thymusdrüse. Die rechte Lunge war im Gegensatze zu der linken normal ausgebildet und zeigte eine ausgesprochene Lappenbildung.

Diese Dislocation der Brusteingeweide war bedingt durch den Eintritt eines grossen Theiles der Baucheingeweide in die Brusthöhle. Diese bestanden aus einem grossen Theile des linken 
Leberlappens, der von der Leber deutlich durch eine Furche abgeschnürt war, ferner aus dem Magen, dem ganzen Convolute der Schlingen des Dünndarmes, dem Coecum, dem Colon ascendens, transversum und dem grösseren Theile des Colon descendens und der sehr stark entwickelten Milz. Die Lage der Eingeweide zu einander war folgende: Der grösste Theil der Bauch-

Fig. 1.

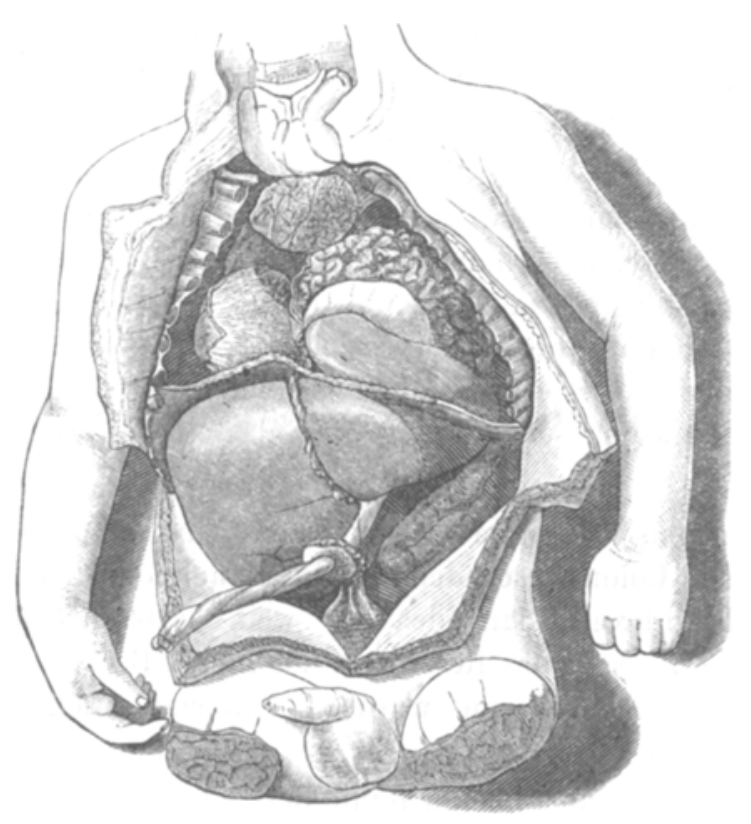

höhle wurde von der massig ausgebildeten Leber eingenommen, welche einen erheblichen Theil ihres linken Lappens durch den Zwerchfellsdefect in den Thoraxraum entsandte. Dieser Lebertheil war von der Hauptmasse des Organes durch eine tiefe Furche getrennt, welche offenbar dadurch entstanden war, dass infolge der Compression der Leber durch das Zwerchfell und die übrigen ausgetretenen Organe an der Stelle der Anlagerung an das Zwerchfell die Entwickelung des Gewebes gehemmt war. Der in dem Brustraume gelegene Theil des linken Leberlappens hatte sich auf dem Zwerchfelle nach vorn umgelegt und füllte den ganzen unteren Theil des linken Thoraxraumes aus. Ueber diesem Leberlappen und hinter demselben lag der Magen, mit seiner nach oben und vorn gewendeten grossen Curvatur über die Leber empor- 
ragend. Der Oesophagus war in normaler Weise durch das Foramen oesophageum in die Bauchhöhle gelangt, hatte sich aber dann um den äusseren Schenkel der das Foramen oesophageum bildenden Muskelpartie herum zurück in die Brusthöhle und zur Cardia des Magens gewendet. Die Cardia war nur wenig von ihrem normalen Platze entfernt, dagegen war der Pylorus stark nach rechts gegen die Cardia zu gedrängt und von dieser nur $1 \mathrm{~cm}$ weit entfernt und mit derselben in gleicher Höhe stehend.

Das Duodenum befand sich in seinem Anfangstheile noch in der Brusthöhle, wendete sich aber dann gegen den Ductus choledochus zu in die Bauchhöhle, da offenbar Leber und Gallengang infolge ihrer grösseren Fixation dem allgemeinen Zuge in die Brusthöhle weniger gut folgen konnten, als der leicht bewegliche Darm. Von der Einmündungsstelle des Ductus choledochus aus bog das Duodenum wieder in einem scharfen Bogen in den linken Brustraum zurück. Ausserdem zeigte das Duodenum noch die Abnormität, dass es nicht in normaler Weise unter dem Colon transversum durch, sondern eng über dasselbe hinwegzog.

Ueber dem Magen lag das ganze Dünndarmpacket mit dem Colon ascendens und dem Colon transversum. Von diesem aus zog sich das Colon descendens in die Bauchhöhe herab, bildete dort mit der Flexura sigmoidea eine weit herabhängende Schlinge und bog dann gegen das Rectum in das kleine Becken ein. Durch die ausgetretenen Gedärme wurde der übrige Theil des linken Brustraumes ausgefüllt.

Den hinteren und oberen Raum der linken Thoraxhälfte nahm die sehr voluminöse, $6 \mathrm{~cm}$ lange, $4 \mathrm{~cm}$ breite und $2 \mathrm{~cm}$ dicke Milz ein. Dieselbe stand mit der grossen Curvatur des Magens und mit dem Colon an einer Stelle in Verbindung, welche etwa der Uebergangsstelle vom Colon transversum in das Colon descendens entsprach, so dass es schien, als habe der durch die Milz ausgeübte Zug die Dislocation des Magens und Colons und die abnorme Stelling des Magens bedingt.

Die Lücke im Zwerchfelle stand zunächst mit der Menge der durch sie ausgetretenen Organe nicht in rechtem Verhältnisse, sondern konnte, in diesem Verhältnisse beurtheilt, eher klein genannt werden. Sie bildete einen in der hinteren Hälfte des linksseitigen Zwerchfelles gelegenen, $5 \mathrm{~cm}$ langen und $1,5 \mathrm{~cm}$ breiten Spalt, welcher von hinten und aussen nach vorn und innen gerichtet war. Nach hinten und aussen wurde dieser Spalt von der 
zehnten und elften Rippe, nach hinten und innen, sowie nach vorn durch scharfrandige, wohl ausgebildete Muskelzüge begrenzt. An diesen Stellen gingen Peritoneum und Pleura glatt in einander über. Die Durchtrittsöffnungen für den Oesophagus und die grossen Gefässe waren normal ausgebildet und standen mit dem Spalte in keiner Communication. Das Centrum tendineum zeigte

Fig. 2.

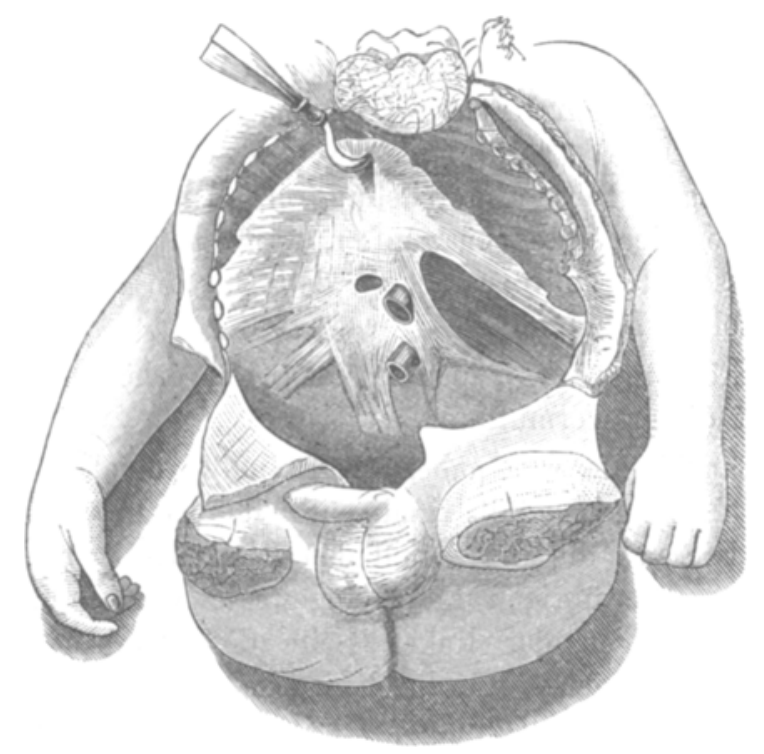

nicht die normale kleeblattähnliche Form, sondern war auf eine kleine, unregelmässige, in der Mitte des Zwerchfelles gelegene Figur zusammengedrängt, so dass dasselbe auch auf der rechten Seite, auf welcher das Zwerchfell im Uebrigen normale Bildung zeigte, fehlerhaft entwickelt war. Der vordere Theil der linken Zwerchfellshälfte war normal entwickelt, auch waren die drei Crura des linksseitigen Lumbaltheiles vollzählig vorhanden, gegen die der rechten Seite jedoch in ihrer Entwickelung beträchtlich zurückstehend. Der Defect war lediglich durch den Wegfall der Muskelzüge der Pars costalis entstanden, welche von der zehnten und elften Rippe ausgehen, dagegen war der Theil der Pars costalis, der an der zwölften Rippe inserirt, sowie der hinterste Theil der an der elften Rippe sich ansetzenden Muskelpartie vollständig erhalten. 
Der zweite der beiden Fälle wurde in der Erlanger Klinik im Juni 1883 bei einer 29jährigen Erstgebärenden beobachtet.

Die Schwangerschaft war vollkommen normal, ebenso die Geburt mit Ausnahme eines übermässig langen Intactbleibens der Fruchtblase, das schliesslich zur Sprengung der Blase Veranlassung gab. Das Kind, ein Knabe, trug alle Zeichen der Reife an sich, kam jedoch stark asphyktisch zur Welt und starb trotz anhaltend angewandter Wiederbelebungsversuche nach $5 / 4$ Stunden. Eine Dislocation des Herzspitzenstosses war dem bei der Geburt anwesenden Arzte nicht aufgefallen, und erst eine von Herrn Prof. $Z$ we ifel an der Leiche vorgenommene percussorische Untersuchung legte die Vermuthung nahe, dass eine Hernia diaphragmatica vorliege. Diese Untersuchung ergab nämlich auf der linken Seite von der ersten bis fünften Rippe herab tympanitischen Percussionsschall, auf der rechten Seite bis an die zweite Rippe hinauf absolute Dämpfung.

Die Eröffnung der beiden grossen Körperhöhlen ergab die Richtigkeit dieser Vermuthung.

Die Bauchhöhle war nahezu vollständig von der enorm vergrösserten Leber ausgefüllt und enthielt ausser derselben nur noch die Nieren, die Blase und den Dickdarm vom Colon descendens an. Die übrigen Baucheingeweide waren durch einen mächtigen Defect in der linken Seite des Diaphragma in die Brusthöhle gedrungen. Hierdurch wurde die linke Lunge stark comprimirt und war vollständig, wie im ersten Falle, nach hinten und oben verdrängt; ausserdem zeigte sie keine Anomalie. Das Herz war über die Mittellinie hinaus nach rechts verdrängt und wirkte seinerseits comprimirend auf die rechte Lunge, von welcher nur der obere Lappen, welcher das Herz etwas überdeckte, lufthaltig war.

Die in den Brustraum frei ausgetretenen Bauchorgane waren: ein grosser Theil des linken Leberlappens, der ganze Magen, der Dünndarm, das Coecum, Colon ascendens und transversum, sowie das Pankreas und die Milz.

Die Leber war beträchtlich vergrössert und maass (am Spirituspräparate gemessen) ohne den in der Brust befindlichen Lappen $14 \mathrm{~cm}$ in der Längè und $6 \mathrm{~cm}$ in der Breite. Ein Theil des linken Lappens hatte sich durch die Oeffnung im Zwerchfelle in den Brustraum geschoben und nahm, indem er auf der ganzen oberen Seite des Diaphragma auflag, das untere Drittel der linken Thorax- 
hälfte fast vollkommen ein. Von der Leber war dieser Lappen durch eine tiefe Furche, die sich an der Stelle und jedenfalls infolge der Anlagerung des Organes an den Zwerchfellrest gebildet hatte, getrennt; seine Länge betrug $5,5 \mathrm{~cm}$, seine Breite $4,7 \mathrm{~cm}$ und seine Höhe 2,8 cm. Die Lage der übrigen in die Brusthöhle ausgetretenen Baucheingeweide war annähernd dieselbe wie im ersten

Fig. 3,

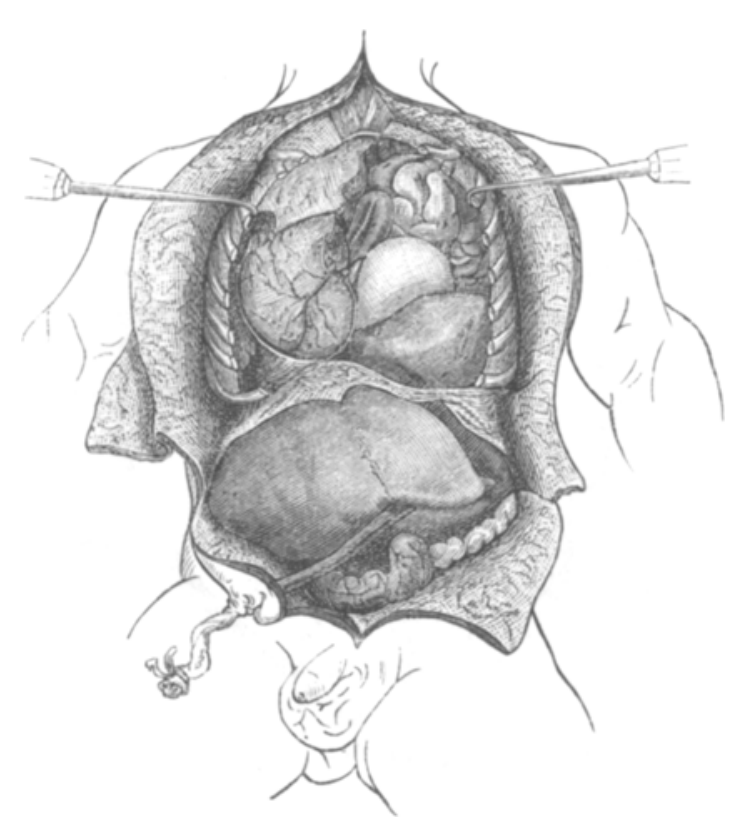

Falle. Der Oesophagus ging ebenfalls durch das normal gebildete Foramen oesophageum in die Bauchhöhle zur Cardia des Magens, welche sich um den äusseren Muskelschenkel dieser Oeffnung hinauf in die Brusthöhle zog. Nach vorn und median von dem Dünndarme, theils neben, theils über dem Magen liegend und über die Mittellinie hinaus in den linken Brustraum hineinragend, befand sich die Milz. Diese hatte eine Länge von $6 \mathrm{~cm}$ und eine grösste Breite von 2,7 cm. Sie war durch eine tiefeindringende Lappenbildung in zwei Lappen getrennt.

Das Herz war über die Mittellinie hinaus nach rechts verdrängt und hatte durch das Andringen der Bauchorgane eine deutliche Drehung nach rechts erfahren. Ueber dem Herzen lag der lufthaltige Oberlappen der rechten Lunge, während der übrige 
444 Bischoff, Drei Fälle von Hernia diaphragmatica congenita.

Theil derselben durch das Herz comprimirt war und nur ein Theil des Unterlappens sich zipfelförmig von der vierten bis zur sechsten Rippe seitlich hineinschob.

Das Zwerchfell war auf der rechten Seite vollständig normal ausgebildet, die Durchtrittsöffnungen für die Aorta descendens und den Oesophagus waren in normaler Weise vorhanden, an

Fig. 4.

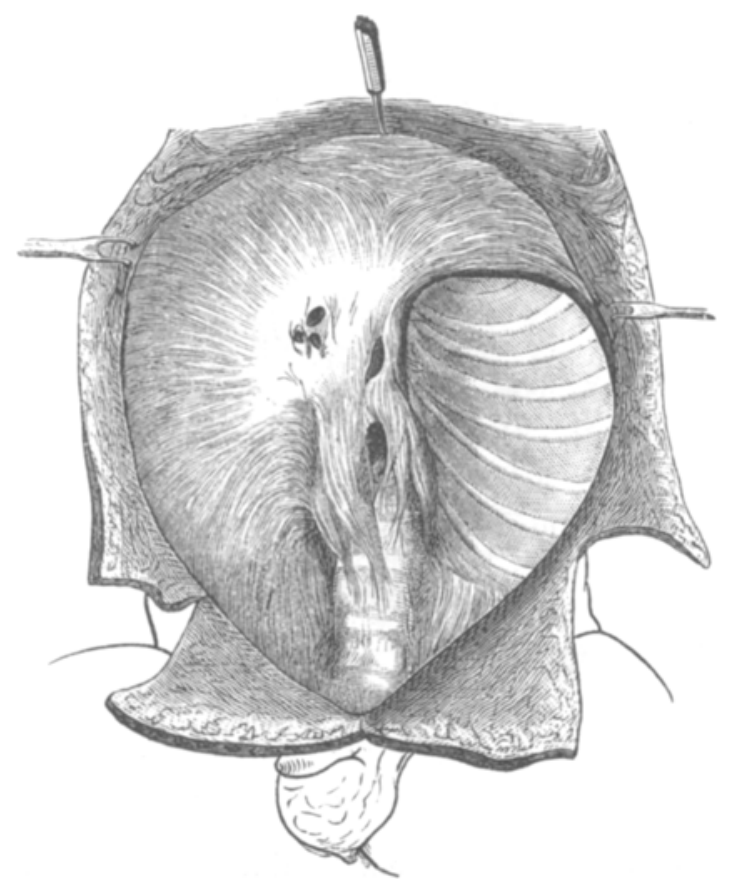

der Stelle des Foramen quadrilaterum befanden sich eine nach vorn liegende grössere und zwei nach hinten liegende kleinere Oeffnungen für die Vena cava ascendens und die noch nicht mit ihr vereinigten Venae hepaticae. Von dem Centrum tendineum war nur der rechtsseitige Theil vorhanden, während der mittlere nur schwach angedeutet war. Linkerseits befand sich in dem Zwerchfelle eine grosse ovale Oeffnung ron $5,5 \mathrm{~cm}$ Tiefe und $4 \mathrm{~cm}$ Breite. Diese Oeffnung wurde nach hinten und aussen durch die neunte bis zwölfte Rippe, nach vorn und aussen durch eine sich nach der Seite hin sichelförmig verjüngende Muskelpartie begrenzt, so dass von der linken Hälfte des Zwerchfelles nur die vom Processus xiphoideus und von der siebenten, achten und ein 
Theil der von der neunten Rippe abgehenden Muskelgruppen vorhanden waren. Die Muskelgruppen der Pars lumbalis waren dagegen vollzählig, wenn auch in geringer Stärke ausgebildet. Der Defect erstreckte sich bis nahe an die Mitte des Zwerchfelles, so dass die Pars tendinea der linken Seite vollständig fehlte; die vorhandene Pars muscularis ging in einem grossen, nach hinten concaven Bogen von der Wirbelsäule zu den Rippen.

Der dritte Fall betrifft einen weiblichen Fötus aus dem neunten Monate. Derselbe, ein Hemikephalus, war im Februar dieses Jahres ron einem auswärtigen Arzte der Anstalt als Demonstrationsobject zugesandt worden. Ausser der Hemikephalie war an demselben noch eine am Kopfe angewachsene Placenta und eine Verkümmerung fast aller Extremitäten ịfolge von Anheftung durch amniotische Fäden, sowie eine mangelhafte Bildung des Gesichtes zu beobachten.

Von den Daten der Schwangerschaft und der Geburt ist nichts Näheres zu erfahren gewesen.

Bei der Eröffnung des Abdomen fand sich in demselben nur der grössere Theil der Leber, die Nieren und das Rectum mit der Flexura sigmoidea und dem Colon descendens. Die sämmtlichen übrigen Organe waren durch eine grosse Oeffnung in der linken Seite des Zwerchfelles in die Thoraxhöhle gedrungen. Infolge dessen war das Herz mit dem Herzbeutel ganz nach rechts gedrängt und verschob seinerseits die ziemlich normal ausgebildete rechte Lunge nach hinten. In der linken Seite des Thoraxraumes befand sich $\mathrm{zu}$ unterst ein $4 \mathrm{~cm}$ hoher und ebenso breiter Theil des linken Leberlappens, über welchem das ganze Convolut der Dünndarmschlingen gelagert war. Schob man dieses nach aussen, so wurde die nur rudimentär ausgebildete linke Lunge sichtbar; die Milz, welche deutlich embryonale Lappung zeigte, und der Magen lagen mehr nach hinten und unten. Der Oesophagus trat durch das vorhandene Foramen oesophageum zur Cardia des Magens, welche annähernd an ihrer normalen Stelle sich befand. Der Magen jedoch wand sich um einen schmalen, die genannte Oeffnung bildenden Muskelzug, nach aufwärts in die linke Brusthöhle.

Die Leber zeigte, mit dem dislocirten Stücke gemessen, eine Länge von $11 \mathrm{~cm}$ und eine Breite von $5,5 \mathrm{~cm}$. Der linke Leberlappen war durch eine auf seiner oberen Fläche befindliche Einkerbung in zwei ungleiche Theile getheilt, von welchen der grös- 
sere in die Brusthöhle gedrungen war und in der Weise daselbst sich umgelegt hatte, dass seine obere Fläche direct nach vorn, die hintere direct nach hinten gerichtet war.

Der Defect nahm in diesem Falle nahezu die ganze linke Hälfte des Zwerchfelles ein, so dass vorn nur ein ganz schmaler, sichelförmiger Rand sichtbar blieb. Die Pars lumbalis war auch hier linkerseits vorhanden, wenn auch schwächer ausgebildet als auf der rechten Seite. Der Muskelzug, welcher das Foramen oesophageum bildete, war durch den nach oben gelagerten Magen in einer weiten Schlinge in die Höhe gezogen; an den übrigen Stellen gingen die Pleura und das Peritoneum glatt in einander über. Die Oeffnung für die Gefässe und den Oesophagus waren vorhanden, das Centrum tendineum in der Mitte und auf der rechten Seite in normaler Weise ausgebildet.

Es zeigen somit alle drei Fälle eine überaus grosse Aehnlichkeit. Es ist nicht nur in allen drei Fällen die Masse der in den Brustraum ausgetretenen Unterleibsorgane annähernd dieselbe, sondern es zeigen auch, worauf es mir am meisten anzukommen scheint, die Lücken im Zwerchfelle eine sehr grosse Gleichheit. Die räumliche Ausdehnung des Spaltes ist zwar eine verschiedene, namentlich handelt es sich im ersten Falle um eine kleinere, mehr spaltförmige Oeffnung, welche mit Ausnahme einer kleinen Stelle überall von Muskelgewebe umgeben ist, während in den beiden anderen der Defect nahezu die ganze linke Seite einnimmt und von der linken Hälfte des Zwerchfelles nur die etwas verkümmerte Pars lumbalis und ein vorderes sichelförmiges Rudiment der Pars costalis gebildet ist.

In allen drei Fällen sind ferner in gleicher Weise die Durchtrittsöffnungen für die grossen Gefässe und den Oesophagus vollständig ausgebildet. Einen besonderen Werth glaube ich darauf legen zu müssen, dass in keinem der drei Fälle der Defect die Mittellinie überschreitet und dass derselbe immer am hinteren Theile der linken Zwerchfellshälfte seinen Sitz hat. In dem letzten Falle ist allerdings auch von der vorderen Partie nur ein ganz geringer Theil ausgebildet. Ausserdem haben die drei Fälle auch das gemeinsam, dass die Lücke nur die Pars costalis betrifft, dass dagegen die Pars lumbalis immer, wenn auch mit der rechtsseitigen Pars lumbalis verglichen, in geringerer Ausbildung vorhanden ist.

Da es sich in allen drei Fällen um eine Hernia diaphragma- 
tica handelt, welche sofort post partum den Tod des Kindes zur Folge hatte, und da in den ersten beiden fast sicher und in dem letzten mit Wahrscheinlichkeit die Entstehung durch ein Trauma auf den graviden Uterus ausgeschlossen werden kann, so bleibt nur noch die Annahme übrig, dass die Missbildung eine Folge fehlerhafter oder vielmehr gestörter Entwickelung des Zwerchfelles sein müsse.

Nach Waldeyer ${ }^{1}$ ) ist die Entwickelung des Zwerchfelles eine paarige. Die Anlage des Diaphragma besteht aus einem centralen und einem dorsalen Theile, und zwar ist der erstere durch das Septum transversum gebildet, welches durch sein Wachsthum das primäre Coelom in die Pericardialhöhle und in die gemeinsame, von Waldeyer das secundäre Coelom genannte Pleuroperitonealhöhle theilt. Der dorsale Theil der ersten Zwerchfellsanlage besteht aus drei Theilen, von denen der eine, der mittlere, dadurch entsteht, dass der centrale Theil an der Rückenfläche der Leberanlage nach abwärts umbiegt, während die beiden anderen, der hintere und der laterale Theil, aus dem die Venae omphalo-mesentericae begleitenden Gewebe abstammen. Von diesem von der Seite in den Pleuroperitonealraum vorspringenden Gewebe gehen nun wiederum nach abwärts zwei Pfeiler aus, welche Waldeyer mit den beiden Gaumenbögen vergleicht, durch deren Zusammenwachsen die vollständige Scheidung zwischen Pleurahöhle und Peritonealhöhle zu Stande kommt.

Es ist in der Publication Waldeyer's schon erwähnt, dass eine congenitale Zwerchfellshernie zu einer Zeit entstehen müsse, in welcher der Abschluss zwischen Pleura- und Peritonealhöhle noch nicht erfolgt sei, wo die offene Stelle noch eine möglichst grosse Ausdehnung besitze, also etwa zu einer Zeit, in welcher jene beiden, mit den Gaumenbögen verglichenen Pfeiler noch nicht gebildet seien.

Es erscheint beim Durchsehen der einschlägigen Literatur und beim Vergleichen der Beschreibungen der Zwerchfellsdefecte diese Annahme als vollständig bestätigt.

In den drei oben beschriebenen Fällen muss in der That in jener Zeit eine Dislocation der Baucheingeweide, wie es scheint, zunächst des linken Leberlappens, vor sich gegangen sein. Das in allen drei Fällen vorhandene sichelförmige vordere Rudiment der

1) Deutsche medicinische Wochenschrift 1884, X. Jahrgang, Nr. 14 , 
Pars costalis würde der Ventralanlage, die ebenfalls überall vorhandene Pars lumbalis der Dorsalanlage des Zwerchfelles entsprechen; letztere ist offenbar infolge des auf sie einwirkenden Druckes von Seiten der Baucheingeweide in ihrer Entwicklung gehemmt worden und steht daher an Masse hinter der Dorsalanlage der rechten Seite beträchtlich zurück.

Im ersten Falle scheint der Austritt der Baucheingeweide später erfolgt zu sein, als in den beiden anderen, da hier der hinterste Theil der Pars costalis des Zwerchfelles, der den beiden anderen vollständig fehlt, vorhanden ist, so dass hier bereits der Abschluss des Zwerchfelles durch das Hereinwachsen des die Venae omphalo-mesentericae begleitenden Gewebes vorbereitet war.

Wodurch allerdings der Austritt der Baucheingeweide herbeigeführt wurde, lässt sich wohl nicht mit Bestimmtheit angeben.

Aus der mir zu Gebote gestandenen ziemlich umfangreichen Literatur über die Hernia diaphragmatica werde ich nur solche Fälle zur Vergleichung herbeiziehen, bei welchen die Missbildung unzweifelhaft angeboren war.

Gruber ${ }^{3}$ ) hat vier Fälle von angeborener linksseitiger Zwerchfellshernie veröffentlicht, bei welchen ausdrücklich bemerkt ist, dass vorn ein sichelförmiges Rudiment des Zwerchfells (die ventrale Anlage) und hinten die Pars lumbalis mit der Oeffnung für den Oesophagus und dem Aortenschlitze vorhanden gewesen sei. In allen vier Fällen betheiligt sich die Leber mit einem Theile ihres linken Lappens, der im ersten Falle beinahe den ganzen linken Thoraxraum ausfüllte. Dieselben Verhältnisse in Bezug auf die Oeffnung im Zwerchfelle erwähnt Kohn ${ }^{2}$ ), Thom. Robinson ${ }^{3}$, welche ebenfalls die Leber vergrössert fanden, Hauss$\operatorname{mann}^{4}$ ), Wertheim ${ }^{5}$, Krombholz ${ }^{6}$ ), Pachner ${ }^{7}$, Wey-

1) 1. Bericht über angeborene Zwerchfellsbrüche. Abhandlung der königl. böhmischen Gesellschaft der Wissenschaften, Folge V, Bd. 5. - 2. und 3. Ibid. - 4. Virchow's Archiv, Bd. 47, S. 382.

2) Dissertation über Hernia diaphragmatica congenita. Erlangen 1874.

3) Schmidt's Jahrbücher der Medicin 1864, Bd. 121, S. 336.

4) Monatsschrift für Geburtskunde u. Frauenkrankheiten 1862, Bd. XIX, S. 404 .

5) Ibid. 1836, Bd. III, S. 396.

6) Bei Würth, Dissertation über Zwerchfellsbruch. Würzburg 1847.

7) Oesterreichische Zeitschrift für praktische Heilkunde, Jahrgang VIII, S. 633. Wien 1861. 
land ${ }^{1}$ ), der ausdrücklich eine ungewöhnliche Vergrösserung der Leber hervorhebt, ,welche dën grössten Theil der Bauchhöhle ausfüllte und selbst noch in die linke Brusthöhle ragte," ferner Lacher ${ }^{2}$, Fischer ${ }^{3}$ ) and $\mathrm{M}^{\text {'Clintock. }}{ }^{4}$ )

Schöller5) erwähnt einen Fall von rechtsseitiger Hernia diaphragmatica, wo bei normaler linker Seite rechts neben den Wirbeln ein ovaler Defect vorhanden war. Hierbei war rechts vorn eine halbmondförmige Platte vorhanden, und gerade vor der Knorpelverbindung der rechten zwölften Rippe stieg ein dickes Muskelbündel, welches sich an die linke Hälfte des Diaphragma anlegte, neben der Mitte des ganzen Diaphragma nach vorn hin. Ueber eine diesem Falle ganz ähnliche Erscheinung berichtet Blöst. ${ }^{6}$ ) In beiden Fällen war der rechte Leberlappen in den Thoraxraum ausgetreten und die Leber vergrössert.

Etwas weniger genau beschrieben, aber nach den vorhandenen Angaben doch mit den oben angeführten offenbar im Wesentlichen übereinstimmend findet sich gleichfalls eine Reihe von Fällen vor:

Feiler ${ }^{7}$ ) veröffentlicht einen Fall, bei welchem die linke Hälfte in der Mitte durchbohrt war, $\mathrm{Otto}^{8}$ ) einen mit dem Defecte in der linken Dorsalregion des Zwerchfelles; ähnliche Fälle sind beobachtet von Mecklenburg ${ }^{9}$ ), Widerhöfer ${ }^{10}$ ), Basedow ${ }^{11}$ ), Eiselt ${ }^{12}$ ), Chard of $\mathrm{Wye}^{13}$ ), ferner von Gilman Duveys ${ }^{14}$ ), Lacher ${ }^{15}$ ) und Balfour. ${ }^{16}$ )

An diese Fälle reiht sich eine Anzahl von solchen an, bei

1) Zeitschrift für Geburtshülfe von Siebold, Bd. XII.

2) Deutsches Archiv für klinische Medicin 1880, Bd. XXVII.

3) Dissertation de Hernia diaphragmatica, citirt bei Lacher.

4) Cannstatt's Jahresbericht 1857, Bd. III, S. 266.

5) Bei Würth.

6) Medicinisches Correspondenzblatt bayerischer Aerzte 1846, Nr. 22, citirt bei Würth.

7) Cannstatt's Jahresbericht für die Medicin 1857, Bd. III, S. 265.

8) In Virchow's Archiv, Bd. 47, S. 393.

9) Vierteljahrsschrift für gerichtliche u. öffentl. Medicin 1855, Bd. VII.

10) Schmidt's Jahrbücher der Medicin 1859, Bd. 104, S. 191.

11) Bei Würth.

12) Ibid.

13) Schmidt's Jahrbücher, Bd. 121, S. 337 citirt.

14) Citirt bei Lacher, Archiv für klinische Medicin 27, 1880.

15) Archiv für klinische Medicin 27, 1880.

16) Ibid. citirt.

Arohiv f. Gynäkologie. Bd, XXV. Hft. 3. 
welchen die Oeffnung nur klein und von Muskelsubstanz überall umgeben war, bei welchen demnach die Defectbildung erst zu einer Zeit begonnen hatte, in welcher der definitive Abschluss der Trennung von Pleura- und Peritonealhöhle schon vorbereitet war.

W. Gruber ${ }^{1}$ ) erwähnt einen solchen Fall, in welchem sich nach hinten und aussen vom linken Sehnentheile eine kreisrunde Oeffnung befand; Fleury ${ }^{2}$ ) fand eine solche, ziemlich enge Oeffnung im Niveau des Foramen oesophageum, ebenso $\mathrm{Kohn}^{3}$ ), $\mathrm{Pe}-$ ters $\left.{ }^{4}\right)$, H. Smith $^{5}$ ), Bailey ${ }^{6}$ ) und $\mathrm{Orth}^{7}$ ). Auf der rechten Seite wurde der Defect in ähnlicher Weise von Duguet ${ }^{8}$ ), Hillier ${ }^{9}$ ), Casper ${ }^{10}$ ) und Houelet Dupuy ${ }^{11}$ ) aufgefunden. Hierher lässt sich vielleicht auch der Fall von Diemenbröck ${ }^{12}$ ) rechnen, in welchem bei einem siebenjährigen Knaben, welcher immer an Asthma gelitten hatte, ein Mangel des sehnigen Theiles beider Zwerchfellshälften aufgefunden wurde.

Breisky ${ }^{13}$ ) und $\mathrm{Busch}^{14}$ ) berichten dagegen über zwei Fälle, bei welchen die Bildung dés Defectes offenbar in früherer Zeit eingetreten war. In dem Falle von Breisky fand sich von dem vorderen Theile der linken Zwerchfellshälfte ein an den Rippen herumlaufender, spitzig endigender Muskelzug, ebenso wie in den drei oben beschriebenen Fällen, dagegen war von der Pars lumbalis nur ein schmaler Muskelzug vorhanden, und es fehlte das Foramen oesophageum und der Aortenschlitz. Busch fand die linke Hälfte des Zwerchfelles vollständig fehlend bis auf ein kleines Rudiment der Pars lumbalis, das zwar den Hiatus aorticus bildete, das Foramen oesophageum dagegen ungebildet liess. Auch in diesem Falle wird die Leber als excessiv vergrössert bezeichnet.

Ueber einen Mangel der ganzen linken Hälfte des Diaphragma berichtet $V$ etter ${ }^{15}$ ), Anthony ${ }^{16}$ ), Destrès ${ }^{17}$ ) und Dreifuss ${ }^{18}$ ),

1) Abhandlung der k. böhmischen Gesellschaft der Wissenschaften, Folge V, Bd. 5 .

2) Citirt Cannstatt's Jahresbericht für die Medicin 1857, Bd. 3.

3) Dissertation über Hernia diaphragmatica congenita. Erlangen 1879. 4-12) Citirt bei Lacher.

13) Vierteljahrsschrift für praktische Heilkunde 1859, Bd. 63, S. 184.

14) Neue Zeitschrift für praktische Geburtskunde von $B u s c h$, d'Outrepont und Ritgen 1837; Bd. V, S. 282.

15) Aphorismen aus der pathologischen Anatomie, S. 145. Wien 1803.

16) Bei Würth.

17) u. 18) Bei Lacher citirt. 
über einen solchen der rechten Seite St. Hilaire' ${ }^{1}$ ); Spessa²) fand einen Fall mit Mangel des ganzen Zwerchfelles.

Es ist in der Literatur noch eine weitere Anzahl unzweifelhaft congenitaler Fälle aufzufinden, die ich aber nicht in den Bereich meiner Vergleichung hereinziehen zu dürfen glaubte, weil bei denselben die Angaben über die Stelle und die Gestaltung des Defectes so allgemein gehalten sind, dass sie ein Urtheil über die rudimentären Zwerchfellstheile und über das Verhältniss der Bildung der Zwerchfellshernie zu der Entwickelung des Zwerchfelles nicht wohl gestatten. Doch möchte ich es nicht unerwähnt lassen, dass in vielen sowohl dieser als der von mir aufgezählten Fälle die Leber durch mehr oder weniger weitgehende Dislocation eines Theiles ihrer Substanz sich an dem Austritte der Eingeweide in die Brusthöhle betheiligte.

Aus den angefïhrten Fällen scheint mir mit Evidenz hervorzugehen, dass das Zustandekommen eines angeborenen Zwerchfellsdefectes durch eine vor dem völligen Verschlusse des Zwerchfelles eingetretene Ausdehnung eines Theiles der Baucheingeweide gegen die spätere Pleurahöhle hin bedingt sei, und dass die Zeit, in welcher dieses Dazwischentreten zu Stande kommt, auf die Ausdehnung des Defectes und damit auf die Grösse der Hernie von direetem Einflusse sei.

In allen eben aufgezählten Fällen, in welchen nicht ein vollständiger Ausfall des Zwerchfelles oder einer Seite desselben beobachtet ist, wurde das Vorhandensein des vorderen Theiles der Pars costalis oder der ganz oder nur theilweise ausgebildeten Pars lumbalis constatirt. Dieser Umstand berechtigt gewiss zu der Annahme, dass in diesen beiden Theilen die erste Anlage des Diaphragma gegeben sei. Ferner scheint mir bewiesen, dass, wenn um die Zeit der Bildung dieser ersten Anlage Baucheingeweide eine Verlagerung in den Thoraxraum erfahren, die weitere Ausbildung des Zwerchfelles dann durch die Dazwischenlagerung dieser Theile gehemmt werde, wenn der Druck ron Seite dieser ebenfalls zunehmenden Eingeweide dem weiteren Wachsthume des Diaphragma unüberwindliche Hindernisse in den Weg legt. Dass in der That ein ziemlich erheblicher gegenseitiger Druck durch das Wachsen der Eingeweide und der vorhandenen Theile

1) Citirt Schmidt's Jahrbücher, Bd. 121, S. 137.

2) Citirt bei Lacher. 
des Diaphragma zu Stande kommt, wird wohl am besten durch die tiefen Druckfurchen bewiesen, welche an der in der Bauchpforte liegenden Leber aufgefunden werden. Der Abschluss der Bildung des Zwerchfelles und die damit gegebene Theilung der Pleuroperitonealhöhle wird offenbar durch ein Gewebe bewerkstelligt, das von den Seiten her gegen die Mitte vorwächst und durch seine schliessliche Verwachsung die noch vorhandene Lücke schliesst. Dieser Schluss kann aus der Betrachtung der Fälle gezogen werden, bei welchen sich ausser dem vorderen Theile der Pars costalis noch weitere, namentlich hintere Theile derselben vorfanden, sowie der Fälle, bei welchen der Defect im Zwerchfelle von allen Seiten von Muskelgewebe eingefasst erschien.

Dass der Defect meist nur auf einer Seite gefunden wird, und in diesem Falle sich strenge auf die eine Seite beschränkt, dürfte wohl als ein Beweis dafür anzusehen sein, dass die Entwicklung des Zwerchfelles eine paarige ist. Fälle, bei welchen auch die Mitte des Diaphragma mit in den Defect hereingezogen ist, sind sehr selten und dürften in der Annahme ihre Erklärung finden, dass das Zwerchfell auf seinen beiden Seiten in seiner Entwicklung gehemmt war.

Mit diesen aus der Vergleichung der einschlägigen Literatur hervorgegangenen Ergebnissen stimmt in geradezu auffallender Weise der Bericht, welchen Waldeyer über die Entwicklungsweise des Zwerchfelles lieferte.

Ueber die Ursache des Eintrittes von Baucheingeweiden in den Brustraum während der fötalen Entwicklung vermag ich allerdings auf Grund dieser Befunde eine befriedigende Erklärung nicht zu geben. Vielleicht dürfte die häufig sich wiederholende, auch von Kohn als auffallend bezeichnete Bemerkung, dass die Leber eine beträchtliche Vergrösserung aufgewiesen habe, sowie die auffallend häufige Betheiligung eines Leberlappen an der Bruchbildung für eine Anzahl von Fällen in einer raschen Volumenzunahme der Leber die erste Ursache für das Zustandekommen einer derartigen Hernie suchen lassen; doch ist so häufig die Leber an der Bruchbildung überhaupt gar nicht betheiligt, dass noch in anderen, unbekannten Factoren die Ursache für das Zustandekommen einer Hernia diaphragmatica gesucht werden muss. 